\title{
FRESHWATER BENTIC DIATOMS IN NILE DELTA AND EL-FAYUM, EGYPT
}

\author{
Ahmed A. El-Awamri \\ Botany Dept., Faculty of Science, Ain Shams Univ., Cairo, Egypt
}

\begin{abstract}
Freshwater Benthic algal samples were collected from seven governorates in the Nile Delta (1- El-Sharkia. 2- El-Gharbia. 3-El-Dakahlya. 4- Damietta. 5-El-Menofia. 6- El-Beheira. 7-Kafr El-Sheikh.) in addition to El-Fayum governorate during the period 2001-2004. The study was made for collecting and identifying as much as possible of the diatom species which occur in the different freshwater benthic habitats. A total of 220 taxa related to 38 genera were identified during this work, 199 taxa from the different localities in Nile Delta and 144 taxa from El-Fayum. The distribution and mean frequencies of diatoms in the sampling localities of the studied area were done. Most of species recorded in this investigation were oligohalobous cosmopolitan. This study showed also that Cyclotella meneghiniana, C. ocellata, Cocconeis placentula, Melosira granulata, Nitzschia palea, N. obtusa and N. obtusa var. scalpelliformis were the most prominent species in Nile Delta and El-Fayum. Ecological data as well as dimensions and photomicrographs of 29 taxa using LM and SEM were made.
\end{abstract}

\section{Keywords: Diatoms, Egypt, Flora, Taxonomy}

\section{Introduction}

Diatoms are distributed in all waters except the hottest and most hypersaline. They are abundant in the phytoplankton and phytobenthos of marine and freshwater whatever the latitude. Hardly a sample can be taken from aquatic habitat that does not contain some cells of diatoms (Round et al., 1990).

In spite of the extensive studies have been carried out in Nile Delta and ElFayum province (El-Ayouty and Ibrahim, 1980, Kobbia et al., 1990, Kobbia et al., 1991, Kobbia et al., 1995, Deyab et al., 2000, Shaaban-Dessouki et al., 2004, AbdelAziz, 2005 and Konsowa et al., 2006). These studies dealt with the distribution and species composition of freshwater algal communities, in relation to the physicochemical characteristics of water as well as in response to some environmental stresses.

Few studies on the freshwater diatom flora in Egypt have been conducted (Muller 1899, Abdin 1949, Hustedt 1949, Aleem 1958, Foged 1980, El-Shimy 1984, Gab-Allah and Touliabah 2000 and El-Awamri et al., 2005.) These studies were restricted in certain geographical regions (River Nile, Sinai, Wadi El-Natrun, El-Fayum and the Greater Cairo). However it is not clear from the literature any taxonomic and floristic studies on freshwater benthic diatoms in Nile Delta. 
Therefore, the study was made for collecting and identifying as much as possible of the diatom species which occur in the different freshwater benthic habitats in Nile Delta and El-Fayum, Egypt.

\section{Materials and Methods}

Benthic freshwater algal samples were collected from seven governorates in the Nile Delta in addition to El-Fayum governorate during the period 2001-2004.

A total of 61 samples from various forms of vegetations and localities were collected. The sample localities were: 1- El-Sharkia (10 samples from Zagazig, Bilbeis and Mania El-Kamh) 2- El-Gharbia (7 samples from Zifta, Kafr El-Ziyat and Tanta). 3 El-Dakahlya (8 samples from Mansoura, Mit Ghamr and Talkha). 4- Damietta (3 samples from Damietta and Kafr El-Batikh). 5-El-Menofia (6 samples from Shibin ElKom, Quesina and El-Bagur). 6-El-Beheira (10 samples from Kafr El-Dawar, Abu Humus and Rosetta) 7-Kafr El-Sheikh (5 samples from Kafr El-Sheikh and Sakha). 8El-Fayum (12 samples from El-Fayum city, Ain El-Celene, Abshway, Sannouris and Tamia). The different forms of algal vegetations include epiphytic diatoms on Ceratophyllum sp. , Lemna gibba, Potamogeton sp. , Cladophora sp., woody Persian wheels, aerophilous flora on mosses, attached diatoms on roots of Phragmites sp., marginal mud, rocks' scraping, small irrigation canals, epipsammic, epilithic and epipelic samples from branches and canals of River Nile and agricultural drains. Relative abundance values for all the collected samples at each governorate were averaged.

The collected samples were prepared for investigation by cleaning frustules using the method described by Jouse et al., (1949-a). The material was mounted according to the method described by Proschkina-Lavernko et al., (1974).

The diatom taxa were identified according to Zabelina et al. (1951), Patrick and Reimer (1966 and 1975), Gasse (1986), Krammer and Lang-Bertalot (1986 and 1988) and Round et al. (1990).

The counting method described by Vilbaste (1994) was applied that 500 valves were counted per sample while the other valves were also identified and recorded. The relative abundance of each taxon was then indicated. In cases where the diatom abundance was low, only 200 valves were counted. Individuals lying on girdle side were also counted.

The collected samples were prepared for LM and SEM investigation by cleaning frustules using the method described by Jouse et al., (1949-b). For LM study, the material was mounted according to the method described by Proschkina-Lavernko 
et al., (1974). The technique used to prepare diatoms for SEM is that adopted by Hasle and Fryxell (1970).

The terminology of the ultrastructure of diatoms follows that suggested by Anonymous (1975), Ross et al., (1979) and Round et al., (1990).

\section{Results and Discussion}

A total of 220 taxa related to 38 genera were identified during this work (from 2001-2004) from Nile Delta and El-Fayum. The distribution and mean frequencies of diatoms in the sampling localities of the studied area were represented in Table (1). Results of the present study revealed that Cyclotella Kützingiana, C. meneghiniana, $C$. ocellata, Cocconeis placentula, C. placentula var.euglypta, Gomphonema parvulum, Melosira granulata, M. granulata var.angustissima, M. varians, Navicula cuspidata, N. mutica, Nitzschia amphibia, N. epiphytica, N. fonticola, N. obtusa, N. obtusa var. scalpelliformis, N. palea, Stephanodiscus hantzschii, Syndera ulna var. danica were the most wide spread taxa in Nile Delta and El-Fayum.

Results also showed that 199 taxa were recorded from the different localities in Nile Delta and 144 taxa were recorded from El-Fayum. Some diatoms taxa were restricted to certain localities, these numbers of taxa ranged from 2-10 in the different localities of Nile Delta, while the exclusive taxa of El-Fayum reached to 29 taxa. This result denotes that the species composition in Nile Delta is similar, cosmopolitan species and varied slightly from the freshwater diatoms of El-Fayum.

By comparison with the diatom assemblages of the different governorates, it was found that the lower number of taxa (48) was recorded in Kafr El-Sheikh while the maximum number was recorded in El-Beheira, This fluctuation mainly due to the number of samples investigated and the nature of habitat of the collected sample (personal observation).

This study showed also that Cyclotella meneghiniana, C. ocellata, Cocconeis placentula, Melosira granulata, Nitzschia palea, N. obtusa, N. obtusa var. scalpelliformis were the most prominent species in Nile Delta and El-Fayum. These taxa were recorded in all sampling localities in relatively high frequencies as compared with the other taxa. The above mentioned species showed some similarities with the diatom flora of River Nile according to the works of (El-Shimy 1984, Gab-Allah \& Touliabah 2000 and El-Awamri et al., 2005.) These similarities qualitatively and some quantitatively especially in the dominance of Cyclotella meneghiniana, C. ocellata and Melosira granulate. Many of the species dominating in one locality may also occur at many or all other investigated localities (cosmopolitan). Thus, it could be concluded that the diatom flora in Nile Delta and El-Fayum differ according to the relative proportions of species than to their presence or absence. Similarly, there were no clear differences in specific diatom composition as function of substrate. For example few of 
the dominant species Cyclotella Kützingiana, C. ocellata, Melosira granulata and $M$. granulata var.angustissima are typically phytoplankton diatoms (Van Heurck, 1899, Hustedt, 1927-1966, Patrick and Reimer, 1966 \& 1975, Foged 1980, Palmer 1980 and Gasse 1986) and represented here as benthic diatoms.

Most of species recorded in this investigation are oligohalobous (freshwater) but there are a group of species such as Achnanthes brevipes, A. brevipes var. intermedia, Amphora coffeaeformis, Bacillaria paradoxa, Biddulphia laevis, Nitzschia hungarica, N. obtusa, N. obtusa var. scalpelliformis N. punctata and Terpsinoe musica which are considered mesohalobous or eurhalyine species ( Hustedt, 1927-1966, Patrick and Reimer, 1966 \& 1975, Foged 1980, and Gasse 1986) were recorded in Nile Delta and El-Fayum in the present investigation and in many Egyptian studies in freshwater habitats (Foged 1980, El-Shimy 1984, Gab-Allah and Touliabah 2000).

In fact and according to these observations, these species have a wide ecological spectrum and therefore they are capable to live in haline as well as freshwater habitats.

\section{Ecological data as well as dimensions and photomicrographs of 29 taxa using LM and SEM. are represented as follows}

\section{Achnanthes brevipes Ag.}

(Plate 1, figs. 1- a\&b)

Dimensions: length $30-110 \mu \mathrm{m}$, breadth $12-28 \mu \mathrm{m}$, striae $7-8$ in $10 \mu \mathrm{m}$

Locality 1,3,4,6 and 8 (epiphytic and epilithic)

Ecology: mesohalobous, but common in Egypt in fresh water habitats.

A. brevipes var. intermedia (Kütz.) Cl. (Plate 1, figs. 2- a\&b).

Dimensions: length $30-110 \mu \mathrm{m}$, breadth $12-28 \mu \mathrm{m}$, striae $7-8$ in $10 \mu \mathrm{m}$

Locality $1,3,4,6$ and 8

Ecology: mesohalobous, but common in Egypt in fresh water habitats.

Anomoneis sphaerophora (kütz) Pfitzer.

(Plate 1, fig. 3).

Dimensions: length $55-65 \mu \mathrm{m}$, breadth $17-20 \mu \mathrm{m}$, striae $12-15$ in $10 \mu \mathrm{m}$

Locality 2,5 and 8

Ecology: halophilic, alkalibiontic, cosmopolitan.

\section{Amphora coffeaeformis (Ag.) Kütz. (Plate 1, fig.4).}

Dimensions: length $26-40 \mu \mathrm{m}$, breadth $10-13 \mu \mathrm{m}$, transapical striae delicate $16-20$ in $10 \mu \mathrm{m}$

Locality: 1 to 8

Ecology: mesohalobous, alkalibiontic, cosmopolitan.

A. ovalis kütz.

(Plate 1, fig. 5).

Dimensions: length $45 \mu \mathrm{m}$, breadth $6 \mu \mathrm{m}$, striae 12 in $10 \mu \mathrm{m}$

Locality: 8

Ecology: oligohalobous (indifferent), alkaliphilic, cosmopolitan. 
Table 1: Mean frequencies of diatoms recorded at the different sample localities 1- ElSharkia. 2- El-Gharbia. 3-El-Dakahlya . 4- Damietta. 5-El-Menofia. 6- El-Beheira. 7-

Kafr El-Sheikh. 8- El-Fayum during 2001-2004.

$P=$ Predominant $(50-20 \%), f=$ frequent $(20-5 \%), c=\operatorname{common}(5-1 \%), r=\operatorname{rare}(1-$

$0.2 \%),+=$ noted and $-=$ not noted

\begin{tabular}{|c|c|c|c|c|c|c|c|c|}
\hline Name of Diatom Taxa & 1 & 2 & 3 & 4 & 5 & 6 & 7 & 8 \\
\hline \multicolumn{9}{|l|}{ Genus: Achnanthes Bory 1832} \\
\hline Achnanthes affinis Grun. & & + & + & + & & + & + & \\
\hline A. brevipes Ag. & + & & + & $\mathbf{r}$ & & $\mathbf{c}$ & & $\mathbf{f}$ \\
\hline A. brevipes var. intermedia (Kütz.) Cl. & + & & + & & & + & & + \\
\hline A. conspicua A. Mayer & & & & & + & & + & \\
\hline A. delicatula (Kütz.) Grun & & + & & $\mathbf{r}$ & & & + & \\
\hline A. exigua Grun. & $\mathbf{r}$ & + & & & & $\mathbf{r}$ & & $\mathbf{r}$ \\
\hline A. hauckiana Grun. & & & + & & & & & + \\
\hline A. hungarica Grun. & & + & & + & & + & & + \\
\hline A. inflata (Kütz.) Grun. & & & & & & & & + \\
\hline A. lanceolata (Bréb.) Grun. & + & & & & & & & + \\
\hline A. marginulata Grun. & & & + & & & + & & \\
\hline A. minutissima var.cryptocephala Grun. & & & & & & + & & + \\
\hline A. orientalis Hust. & & & & & + & & + & \\
\hline \multicolumn{9}{|l|}{ Genus: Amphiprora Ehr. 1841} \\
\hline Amphiprora alata Kütz. & + & & + & & & + & & + \\
\hline \multicolumn{9}{|l|}{ Genus: Amphora Ehr. 1840} \\
\hline Amphora coffeaeformis (Ag.) Kütz & + & + & + & & + & $\mathbf{r}$ & + & + \\
\hline A. ovalis kütz & & & & & & & & + \\
\hline A. ovalis var.pediculus (kütz) V.H.ex Det. & & + & & & + & & & + \\
\hline A. perpusilla Grun. & & & & & & & & + \\
\hline A. turgida Greg. & & & & & & & & + \\
\hline A. veneta kütz & + & & & & & & & + \\
\hline A. ventricosa Greg. & & & & & & & & + \\
\hline \multicolumn{9}{|l|}{ Genus: Anomoneis Pfitzer 1871} \\
\hline Anomoneis sphaerophora (kütz) Pfitzer. & & + & & & + & & & + \\
\hline \multicolumn{9}{|l|}{ Genus: Bacillaria Gmelin 1788} \\
\hline Bacillaria paradoxa Gmelin. & + & & + & & + & + & + & + \\
\hline \multicolumn{9}{|l|}{ Genus: Biddulphia Gray 1821} \\
\hline Biddulphia laevis Ehr. & + & + & & + & & + & & $\mathbf{c}$ \\
\hline \multicolumn{9}{|l|}{ Genus: Caloneis Cl. 1891} \\
\hline Caloneis amphisbaena (Bory.) Cl. & + & + & & + & & & & + \\
\hline C. bacillum (Grun.) Cl. & & & & & + & & & + \\
\hline C. clevei (Lagst.) Cl. & & + & + & & & & + & \\
\hline C.permagna (J.W.Bail.) Cl. & & + & & + & & + & & + \\
\hline C. silicula (Her.) Cl. & + & & & & + & & & + \\
\hline C. silicula var.tumida Hust. & & + & & & + & & & \\
\hline
\end{tabular}


Cont. Table 1

\begin{tabular}{|c|c|c|c|c|c|c|c|c|}
\hline Name of Diatom Taxa & 1 & 2 & 3 & 4 & 5 & 6 & 7 & 8 \\
\hline \multicolumn{9}{|l|}{ Genus: Campylodiscus Ehr. 1840} \\
\hline Campylodiscus clypeus Ehr. & & & + & & + & + & & + \\
\hline C. clypeus var bicostata (W.Sm.) Hüst & & & & & & & & + \\
\hline \multicolumn{9}{|l|}{ Genus: Cocconeis Ehr. 1834} \\
\hline Cocconeis pediculus Ehr. & & & & & & + & & \\
\hline C. placentula Ehr. & c & $\mathbf{f}$ & $\mathbf{p}$ & $\mathbf{r}$ & $\mathbf{f}$ & c & + & $\mathbf{c}$ \\
\hline C. placentula var.euglypta (Ehr.) Cl. & $\mathbf{f}$ & $\mathbf{r}$ & $\mathbf{r}$ & $\mathbf{r}$ & $\mathbf{f}$ & $\mathbf{f}$ & $\mathbf{r}$ & $\mathbf{f}$ \\
\hline C. placentula var. intermedia (Herib.) perag. Cl. & + & + & & + & & & & \\
\hline C. placentula var. roxii (Brun.et perag.) Cl. & & & & & & & & + \\
\hline \multicolumn{9}{|l|}{ Genus: Cyclostephanos Round 1982} \\
\hline Cyclostephanos sp. & & & & & & & & + \\
\hline \multicolumn{9}{|l|}{ Genus: Cyclotella Kütz 1833} \\
\hline Cyclotella comta (Ehr.) Kütz & + & & & + & + & & & \\
\hline C. Kützingiana Thwaites. & $\mathbf{r}$ & $\mathbf{f}$ & $\mathbf{r}$ & $\mathbf{r}$ & + & + & + & + \\
\hline C. meneghiniana Kütz. & c & $\mathbf{p}$ & $\mathbf{f}$ & $\mathbf{c}$ & + & $\mathbf{p}$ & $\mathbf{p}$ & $\mathbf{c}$ \\
\hline C. ocellata Pant. & c & $\mathbf{c}$ & c & $\mathbf{r}$ & $\mathbf{f}$ & $\mathbf{r}$ & $\mathbf{r}$ & $\mathbf{c}$ \\
\hline C. operculata (Ag.) Küitz. & & & + & & & & & + \\
\hline \multicolumn{9}{|l|}{ Genus: Cymatopleura W.Sm. 1851} \\
\hline Cymatopleura elliptica (Bréb.) W.Sm. & & & & + & & + & & + \\
\hline C. solea (Bréb.) W.Sm. & + & + & & & & & & + \\
\hline C. solea var. regula (Ehr.) Grun. & & & & + & & + & & \\
\hline \multicolumn{9}{|l|}{ Genus: Cymbella Ag. 1830} \\
\hline Cymbella affinis Kütz. & + & & & & & & & + \\
\hline C. cistula (Hemp.) Grun & & & & & & + & & \\
\hline C. cymbiformis (Ag.? Kütz.) V.H. & & & & & & & & + \\
\hline C. delicatula Küitz. & & & & + & & & & \\
\hline C. prostrata (Berk) Cl. & & & & & & & & + \\
\hline C.pusilla Grun. & & & & & & + & & \\
\hline C. tumida (Bréb.ex Küitz.) V.H. & + & + & & & + & & + & \\
\hline C. turgida (Greg.) Cl. & & & + & & & & & \\
\hline C. ventricosa Kütz. & & + & & & & + & & + \\
\hline \multicolumn{9}{|l|}{ Genus: Diploneis Ehr. 1840} \\
\hline Diploneis elliptica (Kütz.) Cl. & & & + & & & + & & \\
\hline D. ovalis (Hilse) Cl. & + & + & & + & + & + & & + \\
\hline D. ovalis var. oblongella (Nag.) Cl. & & & + & & & & & \\
\hline \multicolumn{9}{|l|}{ Genus: Epithemia Bréb 1838} \\
\hline Epithemia sorex Kütz. & + & & + & + & & & & + \\
\hline E. turgida (Ehr.) Kütz. & + & & & & + & + & + & + \\
\hline \multicolumn{9}{|l|}{ Genus: Fragilaria Lyngbye 1819} \\
\hline Fragilaria brevistriata Grun. & & & & & & & & + \\
\hline F. brevistriata var. inflata (Pant.) Hüst & & & + & & & & & \\
\hline
\end{tabular}


Cont. Table 1

\begin{tabular}{|c|c|c|c|c|c|c|c|c|}
\hline Name of Diatom Taxa & 1 & 2 & 3 & 4 & 5 & 6 & 7 & 8 \\
\hline F. capucina var.mesolepta Rabh. & + & & & & & & & \\
\hline F. construens (Ehr.) Grun. & & & & + & & & & \\
\hline F. construens var.subsalina Hüst. & & + & & & & + & & \\
\hline F. construens var. venter (Ehr.) Grun. & & & & & & & + & \\
\hline F. crotonensis kitt & & + & & & & & & + \\
\hline F. intermedia Grun. & & & & & & & & + \\
\hline F. lapponica Grun. & & + & & & & & & \\
\hline F. pinnata Ehr. & + & & + & & & & + & \\
\hline \multicolumn{9}{|l|}{ Genus: Frustulia Rabb. 1853} \\
\hline Frustulia. rhomboids (Thwait.) Detoni & & & & & + & & & + \\
\hline F. vulgaris (Thwait.) Detoni & & & + & & & & & + \\
\hline \multicolumn{9}{|l|}{ Genus: Gomphonema Ag. 1824} \\
\hline $\begin{array}{l}\text { Gomphonema acuminatum var.coronatum (Ehr.) } \\
\text { W.Sm. }\end{array}$ & & & & + & & + & & + \\
\hline G. angustatum (Kütz) Rabh. & & & & & & + & & + \\
\hline G. angustatum var.productum Grun. & + & & & & & & & \\
\hline G. augur Her. & & & + & & & & & \\
\hline G. clevei Fricke. & & + & & & & + & & \\
\hline G. constrictum var. capitatum (Ehr.) Cl. & & + & & + & & & & + \\
\hline G. gracile Ehr. & + & & & & & & & \\
\hline G. gracile var. lanceolatum kütz. & & & + & & & + & & \\
\hline G. intricatum Kütz. & + & & & & & & & + \\
\hline G. intricatum var.pumilum Grun. & & & + & & & + & & \\
\hline G. lanceolatum Ehr. & + & & & & & & & \\
\hline G. lanceolatum var.insignis (Greg.)Cl. & + & & & + & & + & & \\
\hline G. longiceps Ehr. & & & + & & & & + & \\
\hline G. longiceps var. subclavata Grun. & & & & & & & & + \\
\hline G. olivaceum (Lyngb.) Kütz. & + & + & & + & & & & + \\
\hline G. parvulum (Kütz.) Grun. & + & + & + & + & + & + & + & + \\
\hline G. parvulum var. lagenulum ( Kütz.Grun.) Hust. & & & + & & & & & \\
\hline G. sphaerophorum Ehr. & & & & + & & & & \\
\hline G. truncatum Ehr. & & & & & & + & & \\
\hline G. sp1 & & & & & & & & + \\
\hline G. sp2 & & & & & & & & + \\
\hline \multicolumn{9}{|l|}{ Genus: Gomphonitzschia Grun. 1868} \\
\hline Gomphonitzschia ungeri Grun. & & + & & + & & & & + \\
\hline \multicolumn{9}{|l|}{ Genus: Gyrosigma Hassall 1845} \\
\hline Gyrosigma acuminatum (Kütz.) Rabh. & & & & & & & & + \\
\hline G. distortum var. parkeri Harr. & & & & & & & & + \\
\hline G. macrum (W.Sm.) Griff \& Henfr. & + & & & & & & & \\
\hline G. spencerii Grun & & & & & + & & & \\
\hline Genus: Hantzschia Grun. 1877 & & & & & & & & \\
\hline
\end{tabular}

Egyptian J. of Phycol. Vol. 7(2), 2006 
Cont. Table 1

\begin{tabular}{|c|c|c|c|c|c|c|c|c|}
\hline Name of Diatom Taxa & 1 & 2 & 3 & 4 & 5 & 6 & 7 & 8 \\
\hline Hantzschia amphioxys (Ehr.) Grun. & + & & + & & + & & + & + \\
\hline H. amphioxys f.capitata O.Müller. & + & + & & + & + & & + & + \\
\hline \multicolumn{9}{|l|}{ Genus: Mastogloia Thw. 1856} \\
\hline Mastogloia braunii Grun & & & & & & + & & + \\
\hline M. elliptica (Ag.) Cl. & & & & & & & & + \\
\hline M. smithi Thw. & & & & & & & & + \\
\hline \multicolumn{9}{|l|}{ Genus: Melosira Ag. 1824} \\
\hline Melosira granulata (Ehr.) Ralfs. & c & $\mathbf{f}$ & c & c & $\mathbf{f}$ & c & $\mathbf{p}$ & $\mathbf{f}$ \\
\hline M. granulata var.angustissima O.Müll. & $\mathbf{f}$ & $\mathbf{f}$ & $\mathbf{r}$ & + & $\mathbf{r}$ & $\mathbf{r}$ & $\mathbf{c}$ & $\mathbf{c}$ \\
\hline M. islandica O. Müll. & & & & & & + & & \\
\hline M. italica (Ehr.) Kütz. & & & & + & & & & + \\
\hline M. varians Ag. & + & $\mathbf{r}$ & $\mathbf{r}$ & + & $\mathbf{f}$ & + & + & $\mathbf{c}$ \\
\hline \multicolumn{9}{|l|}{ Genus: Meridion Ag. 1824} \\
\hline Meridion circulare Ag. & & & & & & & & + \\
\hline \multicolumn{9}{|l|}{ Genus: Navicula Bory 1824} \\
\hline Navicula bacillum Ehr. & & & & & & + & & \\
\hline N. cincta (Ehr.) Kütz. & & & & & & + & & \\
\hline N. confervacea (Kütz.) Grun. & & + & & & & + & + & \\
\hline N. cryptocephala Kütz. & & & & & & & & + \\
\hline N. cryptocephala var. veneta (Kütz.) Grun. & & & & & & & & + \\
\hline N. cuspidata (Küitz.) Cl. & $\mathbf{r}$ & $\mathbf{r}$ & $\mathbf{r}$ & $\mathbf{f}$ & $\mathbf{f}$ & + & + & $\mathbf{f}$ \\
\hline N. cuspidata var.ambigua (Ehr.)Grun. & & & & & & & & + \\
\hline N. dicephala (Ehr.) W.Sm. & & & & & & & & + \\
\hline N. exigua var. capitata Patr. & & & & & & & & + \\
\hline N. gastrum (Ehr.) Kütz. & + & & & & + & & & \\
\hline N. gregaria Donk. & & + & + & & + & + & & + \\
\hline N. halophila (Grun.) Cl. & + & & & + & & + & & + \\
\hline N. menisculus Schum. & + & & + & & + & & + & + \\
\hline N. mutica kütz. & + & + & + & + & + & + & + & + \\
\hline N. pupula Kütz. & & & & & & + & & \\
\hline N.pygmaea Kütz. & + & + & + & + & + & + & & + \\
\hline N. radiosa Kütz. & + & & + & & + & & & + \\
\hline N. rhynchocephala Kütz. & + & + & & + & & & & + \\
\hline N. salinarum Grun. & & & + & & & + & & \\
\hline N. symmetrica Patr. & + & & & & + & & & \\
\hline N. tuscula Her. & & & & & & + & & + \\
\hline N. viridula var.rostellata (Kütz.) Cl. & & + & + & + & & & & + \\
\hline \multicolumn{9}{|l|}{ Genus: Neidium Pfitzer 1871} \\
\hline Neidium affine (Ehr.) Cl. & & & & & & & & + \\
\hline N. dubium (Ehr.) Cl. & & & & & & & & + \\
\hline N. iridis (Ehr.) Cl. & & & & & & + & & \\
\hline
\end{tabular}


Cont. Table 1

\begin{tabular}{|c|c|c|c|c|c|c|c|c|}
\hline Name of Diatom Taxa & 1 & 2 & 3 & 4 & 5 & 6 & 7 & 8 \\
\hline \multicolumn{9}{|l|}{ Genus: Nitzschia Hassall 1845} \\
\hline Nitzschia acicularis W.Sm. & & & & & & & & + \\
\hline N. acuta Hantzsch. & & & & & & & & + \\
\hline N. amphibia Grun. & + & + & + & + & + & + & + & + \\
\hline N. angustata var.acuta Grun. & & & + & & & & & + \\
\hline N. apiculata (Greg.) Grun. & + & & & & & + & & \\
\hline N. bilobata W.Sm. & + & & & + & & & & + \\
\hline N. clausii Hantzsch. & & & & & & & + & \\
\hline N. communis Rabh. & & & & + & & & & \\
\hline N. dissipata (Kütz.) Grun. & & + & & & & + & & + \\
\hline N. epiphytica O. Müll. & + & + & + & + & + & + & + & + \\
\hline N. filiformis (W.Sm.) Hust. & & & & & & + & & + \\
\hline N. fonticola Grun. & + & + & + & + & + & + & + & + \\
\hline N. frustulum (Kütz.) Grun. & + & & & + & + & + & & + \\
\hline N. gracilis Hantzsch. & & & + & & & & & \\
\hline N. granulata Grun. & & & & & & + & & + \\
\hline N. holsatica Hüst. & & & & & + & & & \\
\hline N. hungarica Grun. & + & + & & + & & + & + & + \\
\hline N. intermedia Hantzsch. & & & & & & & & + \\
\hline N. interrupta (Reichelt) Hust. & & + & & & + & + & & + \\
\hline N. kützingiana Hilse. & + & & & & + & & & + \\
\hline N. linearis W.Sm. & + & & & & & + & & \\
\hline N. longissima var. reversa W.Sm. & & & + & + & + & + & & + \\
\hline N. lorenziana Grun. & & & & & + & & & \\
\hline N. microcephala Grun. & & & + & & & & & + \\
\hline N. obtusa W.Sm. & + & + & + & + & + & + & + & + \\
\hline N. obtusa var. scalpelliformis Grun. & $\mathbf{f}$ & $\mathbf{r}$ & $\mathbf{r}$ & $\mathbf{r}$ & + & + & c & $\mathbf{c}$ \\
\hline N. palea (Kütz.) W.Sm. & $\mathbf{f}$ & $\mathbf{r}$ & $\mathbf{r}$ & $\mathbf{r}$ & $\mathbf{f}$ & $\mathbf{f}$ & $\mathbf{f}$ & $\mathbf{r}$ \\
\hline N. paleacea Grun. & & & & & + & & & \\
\hline N. punctata (W.Sm.) Grun. & + & & & + & & & & + \\
\hline N. recta Hantzsch. & + & + & + & & + & & & \\
\hline N. sigma (Kütz.) W.Sm. & & & + & & & & & \\
\hline N. sigmoidea (Ehr.) W.Sm. & + & & + & & + & + & + & \\
\hline N. spetabliis (Ehr.) Ralfs & & + & & + & & & & \\
\hline N. sublineris Hüst & + & & & & + & & & + \\
\hline N. subtilis (Küitz.) Grun. & & + & + & & & & & + \\
\hline N. thermalis Kütz. & + & & & & + & & & \\
\hline N. thermalis var.minor Hilse. & + & & & & & & & \\
\hline N. trybionella Hantzsch. & & & & & & + & & + \\
\hline N. trybionella var. debilis (Arnott) A.Mayer. & & & & & & + & & + \\
\hline N. trybionella var. levidensis (W.Sm.) Grun. & + & & + & & & + & & + \\
\hline
\end{tabular}

Egyptian J. of Phycol. Vol. 7(2), 2006 
Cont. Table 1

\begin{tabular}{|c|c|c|c|c|c|c|c|c|}
\hline Name of Diatom Taxa & 1 & 2 & 3 & 4 & 5 & 6 & 7 & 8 \\
\hline N. trybionella var.victoriae Grun. & & + & + & & & + & + & + \\
\hline N. vitrea Norman. & & & & & + & & & + \\
\hline \multicolumn{9}{|l|}{ Genus:Opephora P. Petit 1888} \\
\hline Opephora martyi Herib. & & & + & & & & & + \\
\hline \multicolumn{9}{|l|}{ Genus: Pinnularia Ehr. 1840} \\
\hline Pinnularia acrosphaeria W.Sm. & + & & + & + & & & & + \\
\hline P. alpina W.Sm. & & & & & & + & & + \\
\hline P. borealis Ehr. & & & & & & & + & + \\
\hline P. braunii (Grun.) Cl. & & & & & + & & & + \\
\hline P. braunii var. amphicephala (A.Mayer) Hust. & & & & & & & + & + \\
\hline P. gibba Ehr. & & + & & & & & & + \\
\hline P. majar (Küitz.) Cl. & & + & & & & & + & + \\
\hline P. microstauron (Ehr.) Cl. & + & + & + & & & + & & + \\
\hline P. microstauron var. brebissonii (Kütz.) Hust. & & & & + & & + & & \\
\hline P. viridis (Nitzsch.) Ehr. & & & + & & + & & & \\
\hline P. viridis var.intermedia $\mathrm{Cl}$. & + & & & & & & & \\
\hline \multicolumn{9}{|l|}{ Genus: Pleurosigma W. Sm. 1852} \\
\hline Pleurosigma angulatum (Quck.) W.Sm. & + & + & & & & & & + \\
\hline P. elongatum W.Sm. & + & & & & + & & + & \\
\hline \multicolumn{9}{|l|}{ Genus: Rhoicosphenia Grun. 1860} \\
\hline Rhoicosphenia curvata (Kütz.) Grun. ex. Rabh. & & + & + & & + & + & & + \\
\hline \multicolumn{9}{|l|}{ Genus: Rhopalodia O. Müller 1897} \\
\hline Rhopalodia gibba (Ehr.) O.Müller. & + & & + & + & + & + & & + \\
\hline R. gibba var. ventricosa (Kütz.) H. \& M. Perag. & & & & & & & & + \\
\hline R. gibberula (Ehr.) O.Müller. & & & + & & & & + & + \\
\hline R. musculus (Kütz.) O.Müller. & & & + & & + & + & & + \\
\hline R. vermicularis O.Müller. & & & & & & & & + \\
\hline \multicolumn{9}{|l|}{ Genus: Stauroneis Ehr. 1843} \\
\hline Stauroneis acuta W.Sm. & & & & & & & & + \\
\hline S. phoenicneteron Ehr. & + & & & + & & + & & \\
\hline \multicolumn{9}{|l|}{ Genus: Stephanodiscus Ehr. 1845} \\
\hline Stephanodiscus astrea (Ehr.) Grun. & & & & & & & & + \\
\hline S. astrea var. minutulus (Kütz.) Cl. et Müll. & + & & + & & & & & + \\
\hline S. hantzschii Grun. & + & + & + & + & + & + & + & + \\
\hline \multicolumn{9}{|l|}{ Genus: Surirella Turpin 1828} \\
\hline Surirella angustata Kütz. & & & & & & & & + \\
\hline S. capronii Bréb. & & + & + & + & & + & + & + \\
\hline S. lapponica A. Cl & + & & & & + & & & \\
\hline S. ovalis Bréb. & + & & & + & & & + & + \\
\hline S. ovata Kütz. & & + & & + & & & & + \\
\hline S. robusta var. splendida Ehr. & & + & & & & + & & \\
\hline
\end{tabular}


Cont. Table 1

\begin{tabular}{|c|c|c|c|c|c|c|c|c|}
\hline Name of Diatom Taxa & 1 & 2 & 3 & 4 & 5 & 6 & 7 & 8 \\
\hline \multicolumn{9}{|l|}{ Genus: Synedra Ehr.1830 } \\
\hline Synedra acus var. radians (Kütz.) Hust. & & & + & & & & & \\
\hline S. capitata Ehr. & & & & + & & & & \\
\hline S. pulchella (Ralfs) Kütz. & & & & & & & + & \\
\hline S. rumpens Kütz. & + & & + & & & & & \\
\hline S. tabulata (Ag.) Kütz. & & & & & & & & + \\
\hline S. ulna (Nitzsch.) Ehr. & + & & + & & & & & $\mathbf{f}$ \\
\hline S. ulna var. aequalis (Kütz.) Hust. & + & + & & & & & & + \\
\hline S. ulna var. danica (Kütz.) Grun. & $\mathbf{r}$ & + & + & + & + & + & + & $\mathbf{f}$ \\
\hline S. ulna var. impressa Hust. & + & & & & & & & \\
\hline S. ulna var.spathulifera Grun. & & & + & & & & & \\
\hline S. vaucheriae Kütz. & & & & & & & + & + \\
\hline \multicolumn{9}{|l|}{ Genus: Terpsinoe Ehr. 1843} \\
\hline Terpsinoe musica Ehr. & & & & & & + & & \\
\hline \multicolumn{9}{|l|}{ Genus: Thalassiosira Cl. 1873} \\
\hline Thalassiosira fluviatilis Hust. & + & & + & & & & & + \\
\hline Number of genera & 27 & 24 & 26 & 22 & 25 & 28 & 19 & 37 \\
\hline Number of taxa & 84 & 65 & 74 & 61 & 62 & 85 & 48 & 144 \\
\hline
\end{tabular}

\section{Bacillaria paradoxa Gmelin.}

(Plate 2, fig. 6).

Dimensions: length $70-80 \mu \mathrm{m}$, breadth 6-8 $\mu \mathrm{m}$, transverse striae $20-25$ in $10 \mu \mathrm{m}$

Keel puncta (fibulae 6-8 in $10 \mu \mathrm{m}$ )

Locality: 1, 3, 5, 6, 7 and 8

Ecology: mesohalobous, $\mathrm{pH}$, indifferent, cosmopolitan, found also in freshwater.

Caloneis silicula (Ehr.) Cl.

(Plate 2, fig.7).

Dimensions: length $55-62 \mu \mathrm{m}$, breadth $12-15 \mu \mathrm{m}$, striae $15-16$ in $10 \mu \mathrm{m}$

Locality: 1,5 and 8

Ecology: oligohalobous (indifferent), alkaliphilic, cosmopolitan.

Cocconeis pediculus Ehr. (Plate 2, figs. 8-a\&b).

Dimensions: length $27-40 \mu \mathrm{m}$, breadth $10-20 \mu \mathrm{m}$, striae $16-18$ in $10 \mu \mathrm{m}$

Locality: 6

Ecology: A frequent epiphyte on aquatic macrophytes. Oligohalobous (in different), alkaliphilic, cosmopolitan.

Cymatopleura elliptica (Bréb.) W.Sm.

(Plate 2, fig. 9).

Dimensions: length $60-68 \mu \mathrm{m}$, breadth $40-45 \mu \mathrm{m}$, marginal costae 4 in $10 \mu \mathrm{m}$

Locality: 4, 6, and 8 .

Ecology: oligohalobous (indifferent), alkaliphilic, cosmopolitan.

C. solea (Bréb.) W.Sm.

(Plate 2, fig. 10).

Dimensions: length $70-95 \mu \mathrm{m}$, breadth $22-25 \mu \mathrm{m}$, marginal costae $8-9$ in $10 \mu \mathrm{m}$

Locality: 1,2 and 8

Ecology: oligohalobous (indifferent), alkaliphilic, cosmopolitan. 
Diploneis ovalis (Hilse) Cl.

(Plate 2, figs. 11- a\&b).

Dimensions: length $30-32 \mu \mathrm{m}$, breadth $12-13 \mu \mathrm{m}$, striae $9-10$ in $10 \mu \mathrm{m}$

Locality: 1, 2, 4, 5, 6 and 8 .

Ecology: oligohalobous (indifferent), alkaliphilic, cosmopolitan.

Gomphonema acuminatum var.coronatum (Ehr.) W.Sm. (Plate 3, fig. 12).

Dimensions: length $30-85 \mu \mathrm{m}$, breadth $7-11 \mu \mathrm{m}$, striae $8-11$ in $10 \mu \mathrm{m}$

Locality: 4, 6 and 8

Ecology: oligotrophic to slightly mesotrophic, cosmopolitan.

G. constrictum var. capitatum (Ehr.) Cl. (Plate 3, fig. 13- a, b and c).

Dimensions: length $30 \mu \mathrm{m}$, breadth $12 \mu \mathrm{m}$, striae $10-11$ in $10 \mu \mathrm{m}$

Locality: 2, 4 and 8.

Ecology: oligohalobous (indifferent), alkaliphilic, cosmopolitan.

G. gracile Ehr.

(Plate 3, fig. 14)

Dimensions: length $45-50 \mu \mathrm{m}$, breadth $9 \mu \mathrm{m}$, striae 12 in $10 \mu \mathrm{m}$

Locality: 1

Ecology: oligohalobous (indifferent), alkaliphilic, cosmopolitan.

G. olivaceum (Lyngb.) Kütz.

(Plate 3, fig. 15).

Dimensions: length $20-35 \mu \mathrm{m}$, breadth $6-8 \mu \mathrm{m}$, striae 12 in $10 \mu \mathrm{m}$

Locality: 1, 2, 4 and 8.

Ecology: oligohalobous (indifferent), alkaliphilic, cosmopolitan.

G. parvulum (Kütz.) Grun.

(Plate3, fig. 16).

Dimensions: length $16-25 \mu \mathrm{m}$, breadth $5-7 \mu \mathrm{m}$, striae $12-14$ in $10 \mu \mathrm{m}$

Locality: 1 to 8

Ecology: oligohalobous (indifferent), $\mathrm{pH}$-circuneutral, cosmopolitan, a wide ecological spectrum.

G. truncatum Ehr.

(Plate 3, fig. 17).

Dimensions: length $40-70 \mu \mathrm{m}$, breadth $8-15 \mu \mathrm{m}$, striae $9-11$ in $10 \mu \mathrm{m}$

Locality: 6

Ecology: oligohalobous (indifferent), alkaliphilic, cosmopolitan, common in shallow freshwater habitats.

Navicula viridula var.rostellata (Kütz.) Cl. (Plate 4, fig.18).

Dimensions: length $30-35 \mu \mathrm{m}$, breadth $9-11 \mu \mathrm{m}$, striae $9-10$ in $10 \mu \mathrm{m}$

Locality: 2, 3, 4 and 8 .

Ecology: oligohalobous (indifferent), alkaliphilic, cosmopolitan.

N. amphibia Grun.

(Plate 4, fig. 19).

Dimensions: length $12-16 \mu \mathrm{m}$, breadth $3-4 \mu \mathrm{m}$, fibulae $7-9$ in $10 \mu \mathrm{m}$

Locality: 1 to 8.

Ecology: oligohalobous (indifferent), alkaliphilic, cosmopolitan.

Nitzschia angustata var.acuta Grun. (Plate 4, fig. 20).

Dimensions: length $31-40 \mu \mathrm{m}$, breadth 5-7 $\mu \mathrm{m}$, transapical striae $18-20$ in $10 \mu \mathrm{m}$

Locality: 3 and 8.

Ecology: oligohalobous (indifferent), alkaliphilic, cosmopolitan. 
N. hungarica Grun.

(Plate 4, fig. 21).

Dimensions: length $70-75 \mu \mathrm{m}$, breadth $6-9 \mu \mathrm{m}$, keel puncta fibulae $7-9$ in $10 \mu \mathrm{m}$, transapical striae $16-20$ in $10 \mu \mathrm{m}$

Locality: 1, 2, 4, 6, 7 and 8 .

Ecology: mesohalobous, alkaliphilic, cosmopolitan, found in freshwater.

N. microcephala Grun.

(Plate 4, fig. 22).

Dimensions: length $8-15 \mu \mathrm{m}$, breadth $3-4 \mu \mathrm{m}$, fibulae $12-13$ in $10 \mu \mathrm{m}$, transapical striae $33-35$ in $10 \mu \mathrm{m}$

Locality: 3 and 8.

Ecology: oligohalobous (indifferent), alkaliphilic, cosmopolitan.

N. obtusa W.Sm.

(Plate 4, figs. 23- a\&b).

Dimensions: length $50-75 \mu \mathrm{m}$, breadth $4-7 \mu \mathrm{m}$, fibulae $9-10$ in $10 \mu \mathrm{m}$, transapical striae 30-32 in $10 \mu \mathrm{m}$

Locality: 1 to 8.

Ecology: oligohalobous (indifferent), alkaliphilic, cosmopolitan.

N. punctata (W.Sm.) Grun.

(Plate 5, figs. 24).

Dimensions: length $9-12 \mu \mathrm{m}$, breadth $5 \mu \mathrm{m}$, transapical striae $10-20$ in $10 \mu \mathrm{m}$

Locality: 1,4 and 8 .

Ecology: eurhaline and mesohalobous, rarely freshwater.

Opephora martyi Herib.

(Plate 5, fig. 25).

Dimensions: length $8-11 \mu \mathrm{m}$, breadth $3-4 \mu \mathrm{m}$, striae $8-9$ in $10 \mu \mathrm{m}$

Locality: 3 and 8

Ecology: oligohalobous (indifferent), alkaliphilic, cosmopolitan.

Surirella ovalis Bréb.

(Plate 5, fig. 26).

Dimensions: length $31 \mu \mathrm{m}$, breadth $20 \mu \mathrm{m}$.

Locality: 1, 4, 7 and 8

Ecology: mesohalobous, cosmopolitan.

S. ovata Kütz.

(Plate 5, fig. 27).

Dimensions: length $30 \mu \mathrm{m}$, breadth $24 \mu \mathrm{m}$.

Locality: 2, 4 and 8

Ecology: oligohalobous (indifferent), alkaliphilic, cosmopolitan.

Synedra tabulata (Ag.) Kütz.

(Plate 5, fig. 28).

Dimensions: length $45 \mu \mathrm{m}$, breadth $2-7 \mu \mathrm{m}$, striae $9-14$ in $10 \mu \mathrm{m}$

Locality: 8

Ecology: slightly brackish, cosmopolitan.

S. ulna var. danica (Kütz.) Grun.

(Plate 5, fig. 29).

Dimensions: length $110-170 \mu \mathrm{m}$, breadth $5-8 \mu \mathrm{m}$, striae $8-10$ in $10 \mu \mathrm{m}$

Locality: 1 to 8

Ecology: oligohalobous (indifferent), alkaliphilic, cosmopolitan. 


\section{References}

Abdel Aziz, S. G. (2005). Study of the water quality of the River Nile, with relation to the environmental condition at El-kanater El-Khyria Region. M. sc. Thesis, Fac. Sci. (Girls), Chemistry Departement, Al-Azhar University, 188 pp.

Abdin, G. (1949): Benthic Algal flora of Aswan Reservoir (Egypt). Hydroboil. 2:118-133.

Aleem, A.A. (1958). A taxonomic and palaeoecological investigation of the diatom flora of the extinct Fayoum Lake (Egypt). I. System. Part 2: Distrib. And Ecol. Bull. Fac, Sc, II:99-138; 217-243. Alexandria Univ. Press.

Anonymous, (1975). Proposal for a standardization of diatom terminology and diagnosis. Nova Hedwigia, 52: 323-354

Deyab, M.A.; Nemat Alla, M.M. and El Adl, M.F. (2000). Phytoplankton as indicator of water quality in the irrigation and drainage canals in westernDamietta-Egypt. Egypt. J. Phycol, 1: 15-31.

El-Awamri, A.F , Shaaban, A.S. and A.I. Saleh (2005). Diatom flora from different Aquatic Habitats in the Greater Cairo (Egypt) Int. J. Argri. Bio. 7(2): 230-39

El-Ayouty, E.Y. and Ibrahim, E.A. (1980). Distribution of phytoplankton along the River Nile. Water Supply and Management, 4: 35-44.

El- Shimi, A.A. (1984). Studies on diatoms of River Nile in A.R.E. Ph. D. Thesis. Univ. Coll. For Girls. Ain Shams Univ., 227 pp.

Foged, N. (1980): Diatoms in Egypt. Nova Hedwigia, 33: 629-707.

Gab-Allah, M.M. and Touliabah, H.E. (2000). Diatom assemblages in superficial sediments from Nasser Lake, Egypt. In: proceedings of the 1st Int. Conf. Biol. Sci. (ICBS); Fac. Sci., Tanta Univ., pp. 89-103.

Gasse, F. (1986). East African Diatoms: taxonomy, ecological distribution. Bibliotheca Diatomologica. Band II. J.Cramer, Berlin, 201 pp.

Hasle, G.R. and Fryxell, G.A. (1970). Diatoms; cleaning and mounting for light and electron microscopy. Trans. Am. Microsc. Soc., 89: 469-474.

Hustedt, F. (1927-1966). Die Kieselalgen Deutschlands, Oester - reichs und der Schweiz Rabenhorst's Kryptogamenflora, VII. Akademisch Verlagsgesellschaft Leipzig. (Cited in Sterrenburg, F.A.S., 1994).

Hustedt, F . (1949). diatomeen von der Sinai-Halinset und aud dem Libanon-Gebiet. Hydrobiologica 5(2): 24.

Jouse, A.P.; Proschkina-Laverenko, A.I. and Sheshykova, V.S. (1949 a). Diatomical Analysis. Vol. 1. Publ. "Geol. Liter". Leningrad, 239 pp.

Jouse, A.P.; Kicelov, I.A.: Proschkina-Laverenko, A.I. and Sheshykova, V.S. (1949 b). Diatomical Analysis. Vol. 2. Publ. "Geol. Liter". Leningrad, pp. 212-213.

Kobbia, I.A.; Shabana, E.F.; Dowidar, A.E. and El-Attar, S.A. (1990). Changes in physico-chemical characters and phytoplankton structure of Nile water in the vicinity of iron and steel factory at Helwan (Egypt). Egypt. J. Bot., 33(3): 215-233. 
Kobbia, I.A.; Hassan, S.K.M. and Shoulkamy, M.A. (1991). Plankton ecology of Bahr Yosef and Ibrahimmya Canals in the vicinity of Minia at Upper Egypt. Bull. Fac. Sci., Cairo Univ., 59: 129-153.

Kobbia, I.A.; Metwali, R. M. and El-Adel, H.M. (1995): Studies on freshwater at Qalubia province (Egypt) in relation to some physico-chemical factors. Egypt. J. Bot., 35(1): 25-43.

Konsowa, A. H., Salah El Din, R. A., El-Awamri, A. A., and Zaher, S. S. (2006). Changes in phytoplankton community structure in relation to different wastes discharging into Rosetta Branch (Egypt). Egyptian J. of Phycol. 7(2): 247-263.

Krammer, K. and Lange-Bertalot, H. (1986). Bacillariophyceae. 1.Teil: Naviculaceae. In: Süsswasserflora von Mitteleuropa. Band 2/1. H. Ettl; J. Gerloff; H. Heynig and D. Mollenhauer (eds.).Gustav Fisher Verlag, Stuttgart, 876 pp.

Krammer, K. and Lange-Bertalot, H. (1988). Bacillariophyceae. 2. Teil: Bacillariaceae, Epithemiaceae and Surirellaceae. In: Süsswasserflora von Mitteleuropa. Band 2/2. H. Ettl; J. Gerloff; H. Heynig and D.Mollenhauer (eds.). Gustav Fisher Verlag, Stuttgart, 821 pp

Muller, O. (1899). Bacillariaceen aus den Natronthälern von El Kab (Oberagypten). Hedwigia 38:274-321. Taf. 10-12.

Palmer, C.M. (1980). Algae and Water Pollution: the identification, significance and control of algae in water supplies and in polluted water. Castle House Publications Ltd, U.K., 124 PP.

Patrick, R. and Reimer, C.W. (1966). The diatoms of the United States (Exclucive of Alaska and Hawaii). Vol. 1: Fragilariaceae, Eunotiaceae, Achnanthaceae, and Naviculaceae. Monogrs. Acad. Sci. Philad. 13: 688 pp.

Patrick, R. and Reimer, C.W. (1975). The diatoms of the United States (Exclucive of Alaska and Hawaii). Vol.2, Part 1: Entomoneidaceae, Cymbellaceae, Gomphonemaceae, and Epithemiaceae. Monogrs. Acad. Sci. Philad. 13: 213 pp.

Proschkina-Laverenko, A.I.; Gleser, S.I.; Jouse, A.P.; Makarova, I.V. and Schesschykova-Poretzaja, V.S. (1974). The Diatoms of USSR. Fossil and Recent- Vol.I. Publ. House“Sov. Nauke.”, Leningrad. Bronch., 372 pp.

Ross. R. Cox, E.J., Karayeva, N.I., Mann. D.G., Paddock, T.B.B., Simonsen. R. and Sims, P.A. (1979). An amended terminology for siliceous components of the diatom cell. Nova Hedwigia, Beih., 64: 513 - 533.

Round, F.E.; Crawford, R.M. and Mann, D.G. (1990). The diatoms: biology and morphology of the genera. Cambridge University Press, Cambridge, 747 pp.

Shaaban- Dessouki, S. A.; Deyab, M. A. and Mofeed, J. (2004). Phycological assessment of water quality of River Nile Delta- Egypt. Egyptian J. of Phycol., 5:18-34.

Van Heurck, H. (1899). Traité des Diatomées. Private edition, Antwerp. (Cited in Sterrenburg, F.A.S., 1994). 
Vilbaste, S. (1994). The epiphytic and microphytobenthic diatoms in Estonian coastal waters (the Baltic Sea). In: Proceedings of the 13th International Diatom Symposium. D. Marino \& M. Montresor (eds.). Biopress Limited, Bristol, pp. 259-269.

Zabelina, M.M.; Kicelov, I.A.; Proschkina-Laverenko, A.I. and Scheshykova, V.S. (1951). The Freshwater Algaeof USSR. Vol.4. Bacillariophyta. Publ. "Sov. Nauk." Moscow, 619 pp.

\section{دياتومات المياه العذبة اللاصقة فى بعض مناطق دلتا النيل و الفيوم (مصر)}

$$
\text { قسم النبات كلبة العلوم ـجامعة عبن شعد الرس }
$$

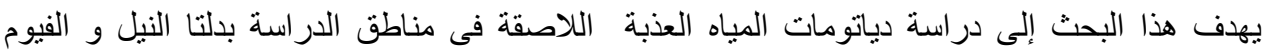

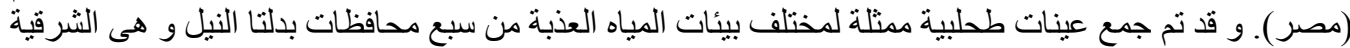

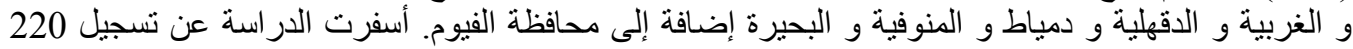

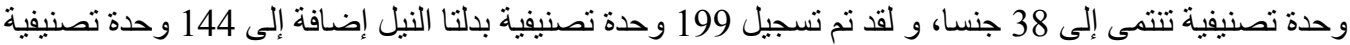

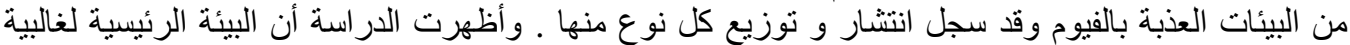

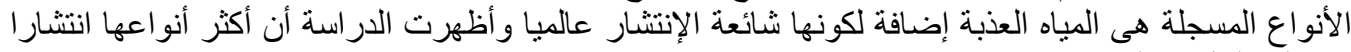

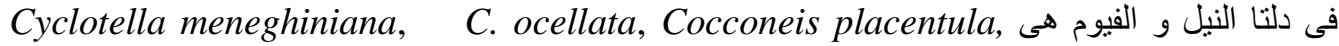
Melosira granulata, Nitzschia palea, N. obtusa, N. obtusa var. scalpelliformis و هذا العمل إثتنمل أيضا على ذكر أهم الظروف البيئية و الظاهرية لعدد 29 وحدة تصنيفية مستعينا بذلك بالمجهر الضوئى و المجهر الإلكترونى الماسح. 

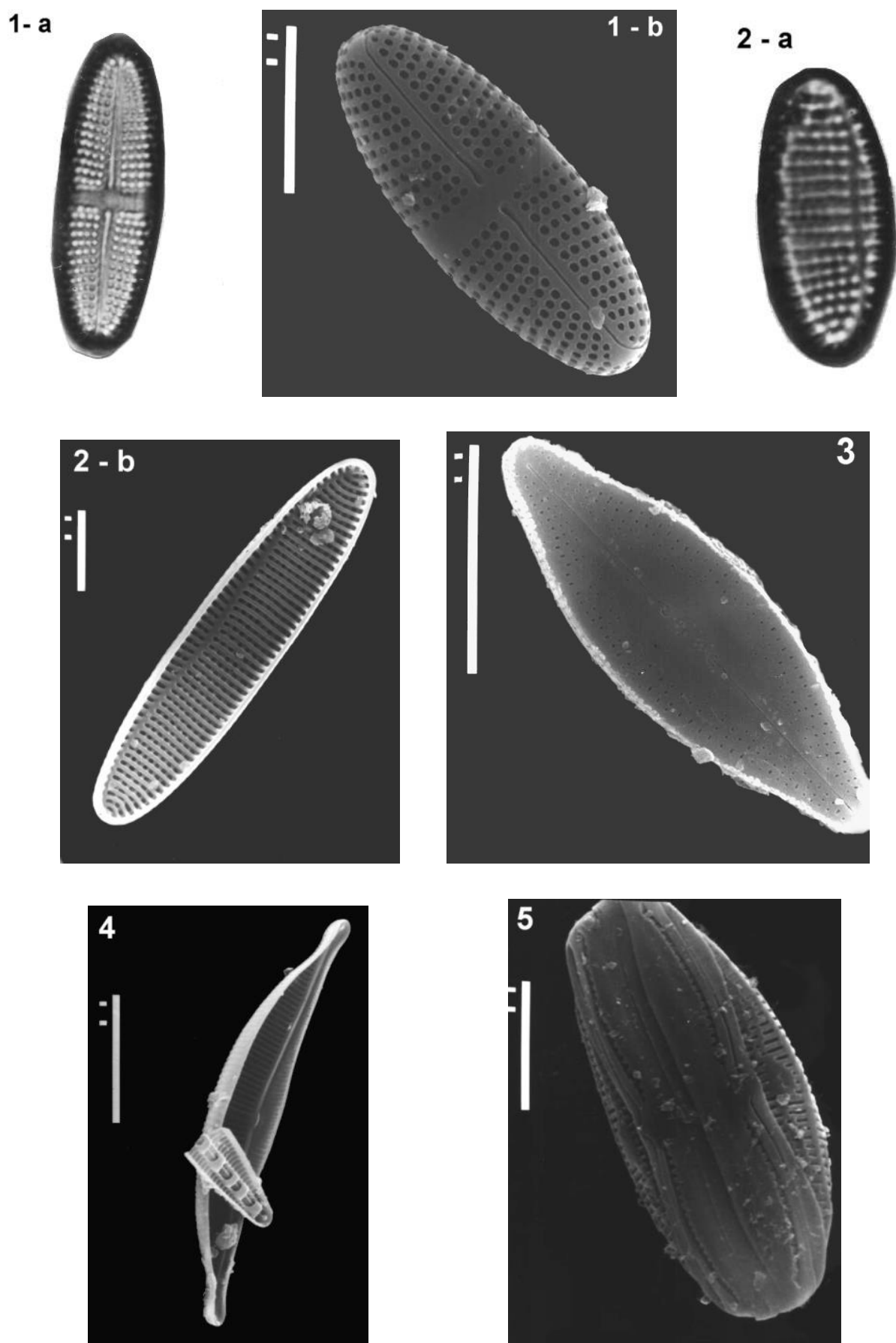

Plate I: Fig.1-a: Achnanthes brevipes Ag. LM(x1500), fig. 1-b: A. brevipes Ag. SEM, bar= $10 \mu \mathrm{m}$, fig. 2-a: A. brevipes var. intermedia (Kütz.) Cl. LM(x1500), fig. 2-b: A. brevipes var. intermedia (Kütz.) Cl. SEM, bar=10 $\mu \mathrm{m}$, fig. 3: Anomoneis sphaerophora (kütz) Pfitzer. SEM, bar $=10 \mu \mathrm{m}$, fig. 4: Amphora coffeaeformis (Ag.) Kütz SEM, bar $=10 \mu \mathrm{m}$, fig. 5: A. ovalis kütz $\mathrm{SEM}$, bar $=10 \mu \mathrm{m}$. 

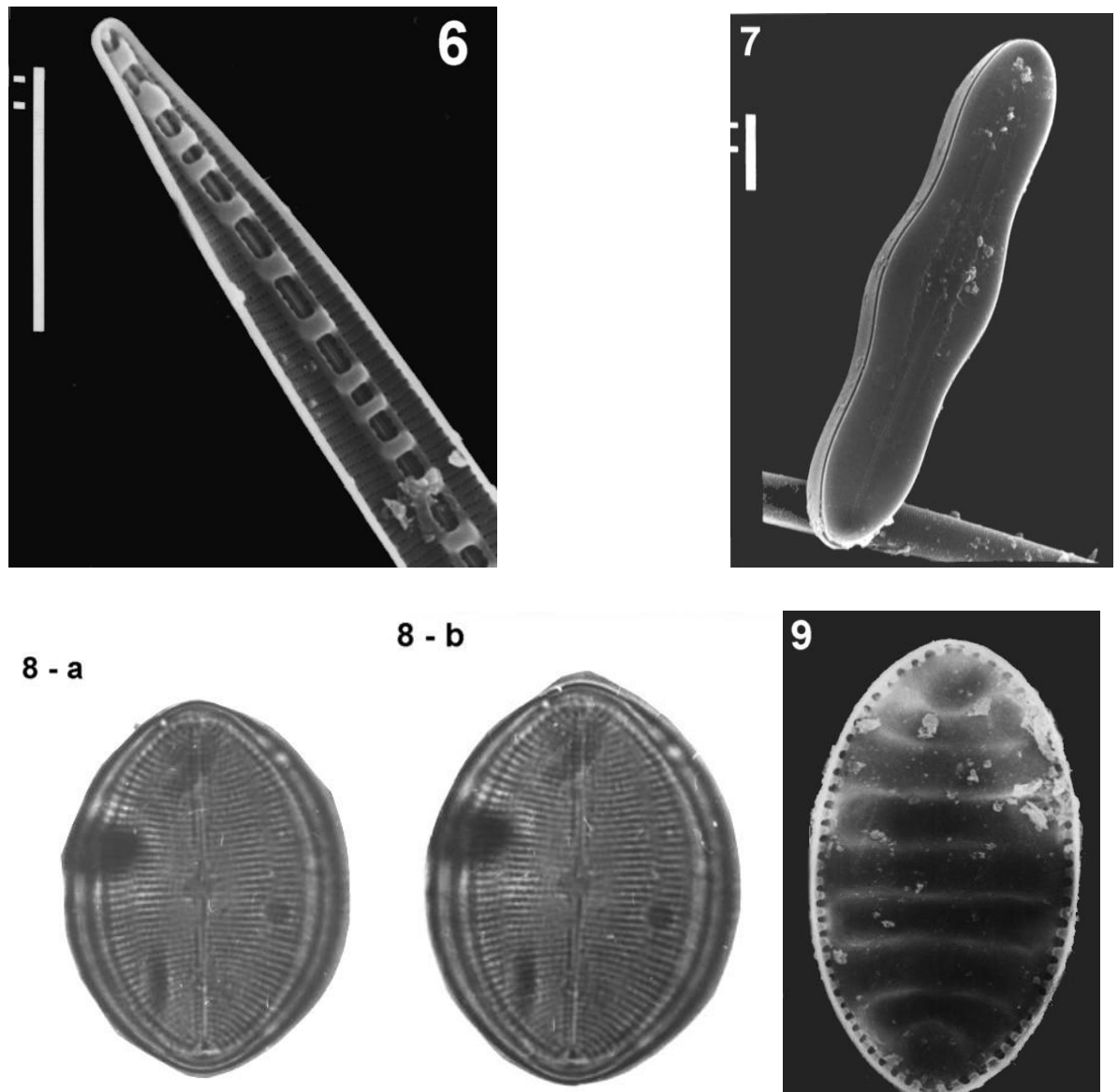

$8-b$
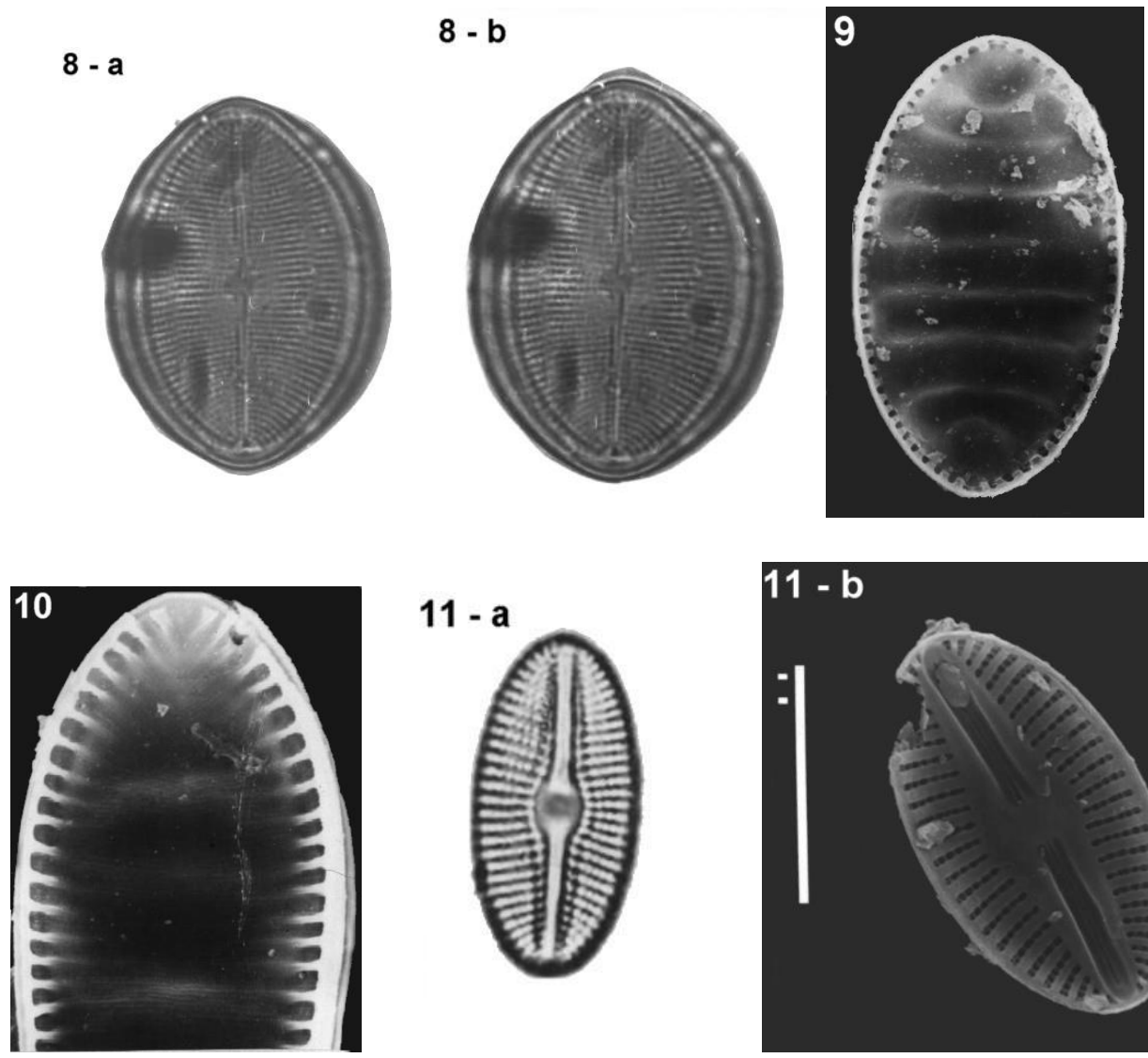

11 - a
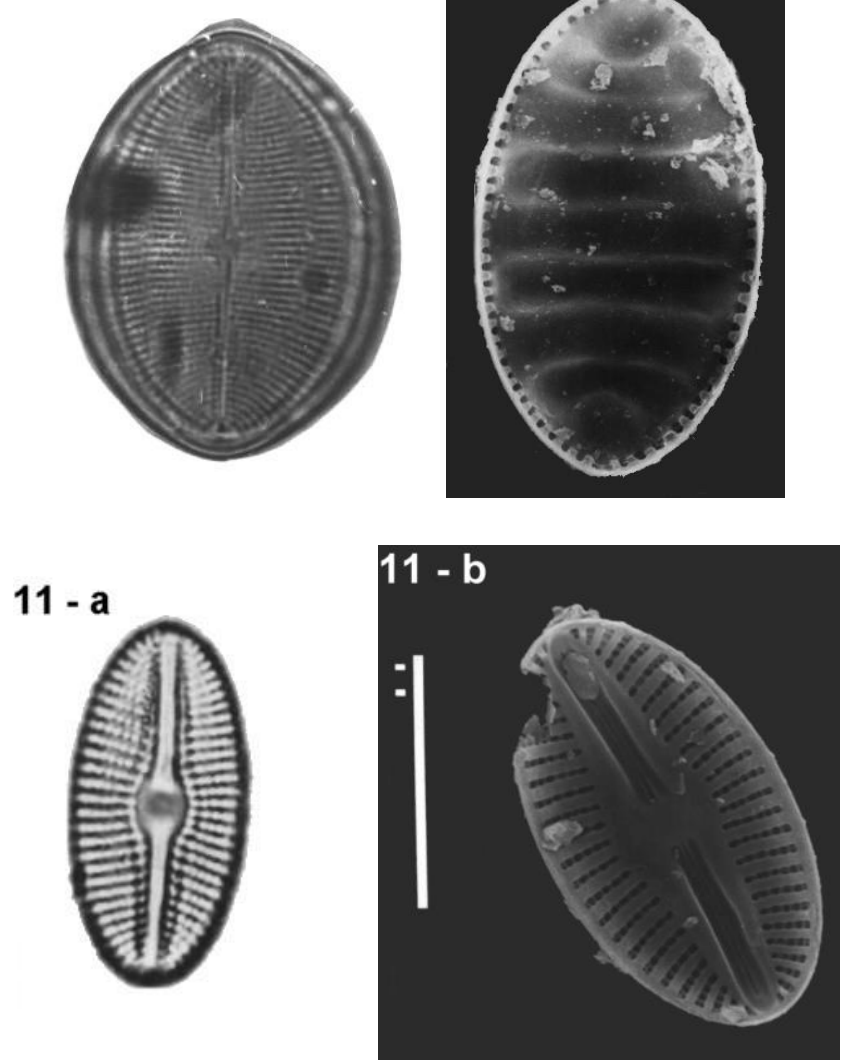

Plate II: Fig. 6: Bacillaria paradoxa Gmelin. SEM, bar $=10 \mu \mathrm{m}$, fig. 7: Caloneis silicula (Ehr.) Cl. SEM, bar $=10 \mu \mathrm{m}$, fig. 8-a: Cocconeis pediculus Ehr. LM(x1000), fig. 8-b: C. pediculus Ehr. LM(x1000), fig. 9: Cymatopleura elliptica (Bréb.) W.Sm. SEM, bar $=10 \mu \mathrm{m}$, fig. 10: C. solea (Bréb.) W.Sm. SEM, bar $=10 \mu \mathrm{m}$, fig. 11-a: Diploneis ovalis (Hilse) Cl. LM(x1500), fig. 11-b: D. ovalis (Hilse) Cl. SEM, bar $=10 \mu \mathrm{m}$. 

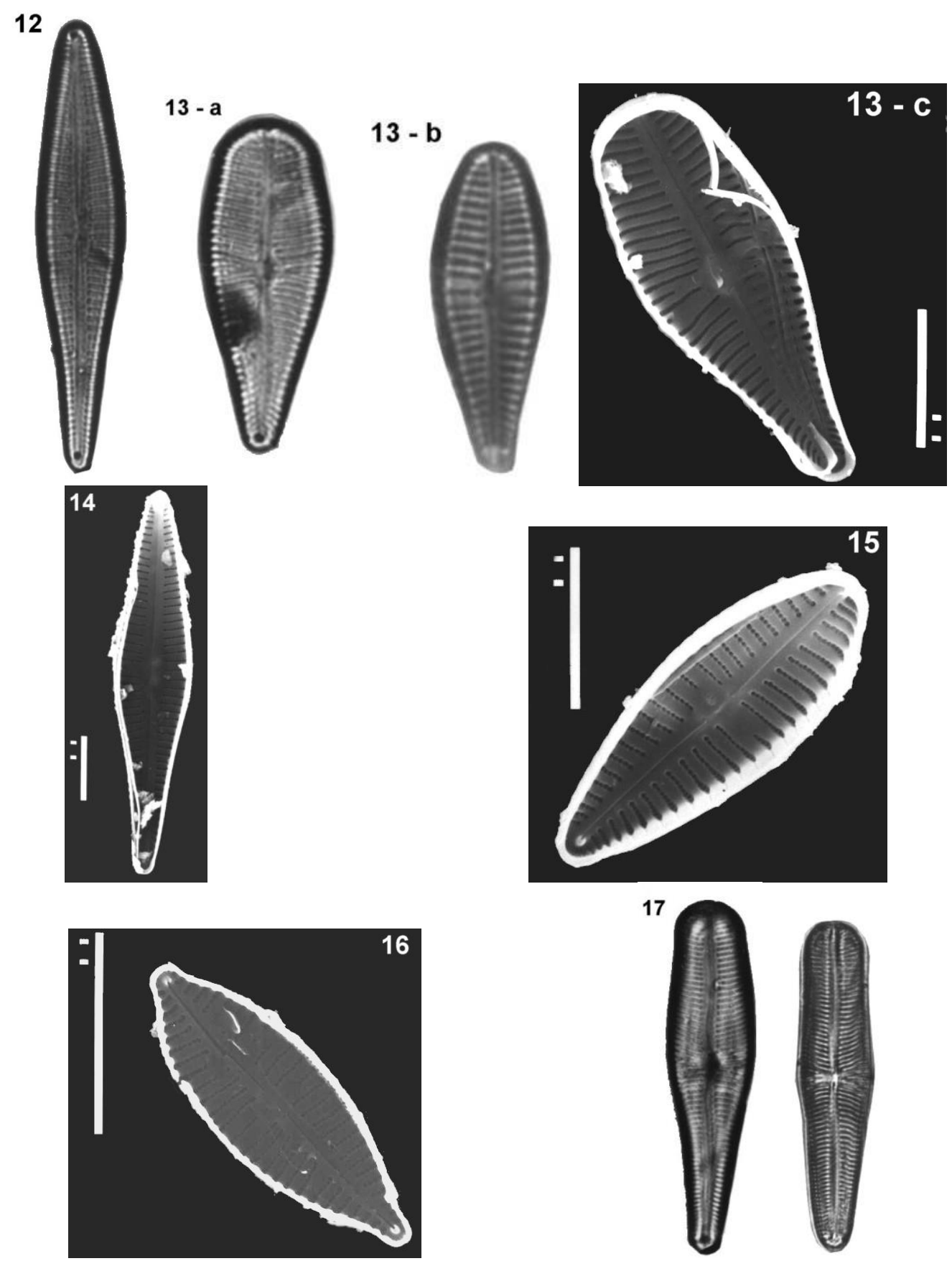

Plate III: Fig. 12: Gomphonema acuminatum var.coronatum (Ehr.) W.Sm. LM(x1500), fig. 13a\&b: G. constrictum var. capitatum (Ehr.) Cl. LM(x1500), fig. 13-c: G. constrictum var. capitatum (Ehr.) Cl. SEM, bar $=10 \mu \mathrm{m}$, fig. 14: G. gracile Ehr. SEM, bar $=10 \mu \mathrm{m}$, fig. 15: G.

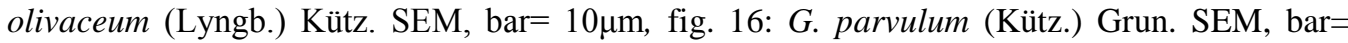
$10 \mu \mathrm{m}$, fig. 17: G. truncatum Ehr. LM(x1000). 
18
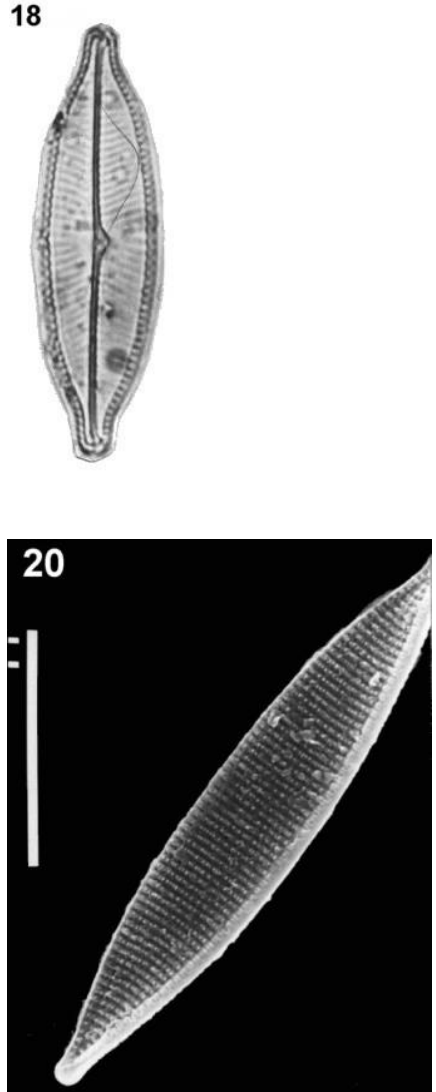

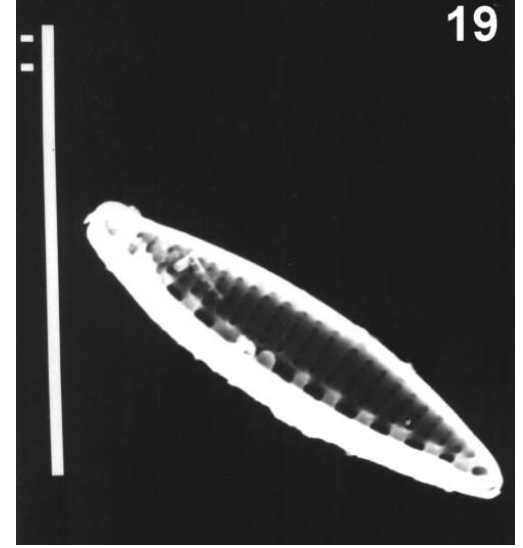

21

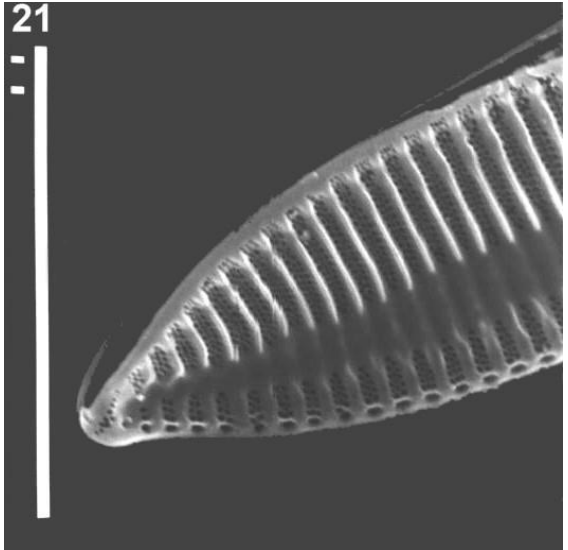

$23-a$
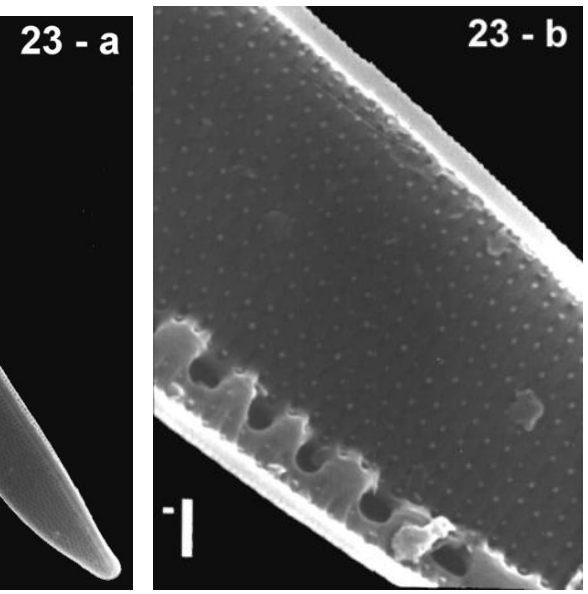

Plate IV: Fig. 18: Navicula viridula var.rostellata (Kütz.) Cl. LM (x1500), fig. 19: N. amphibia Grun. SEM, bar $=10 \mu \mathrm{m}$, fig. 20: Nitzschia angustata var.acuta Grun. SEM, bar $=10 \mu \mathrm{m}$, fig. 21: $N$. hungarica Grun. SEM, bar $=10 \mu \mathrm{m}$, fig. 22: N. microcephala Grun. SEM, bar $=10 \mu \mathrm{m}$, fig. $23-$ a: $N$. obtusa W.Sm. SEM, bar=10 $\mu \mathrm{m}$, fig. 23-b: $N$. obtusa W.Sm. SEM, bar $=1 \mu \mathrm{m}$. 

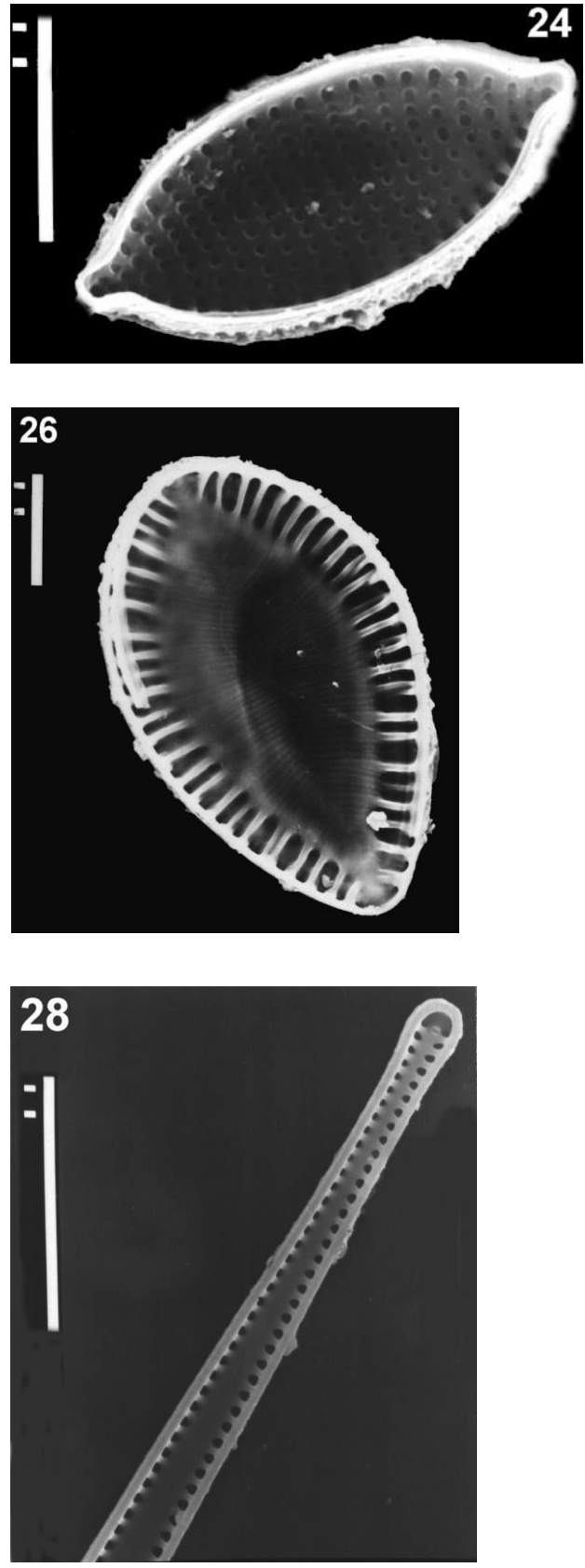
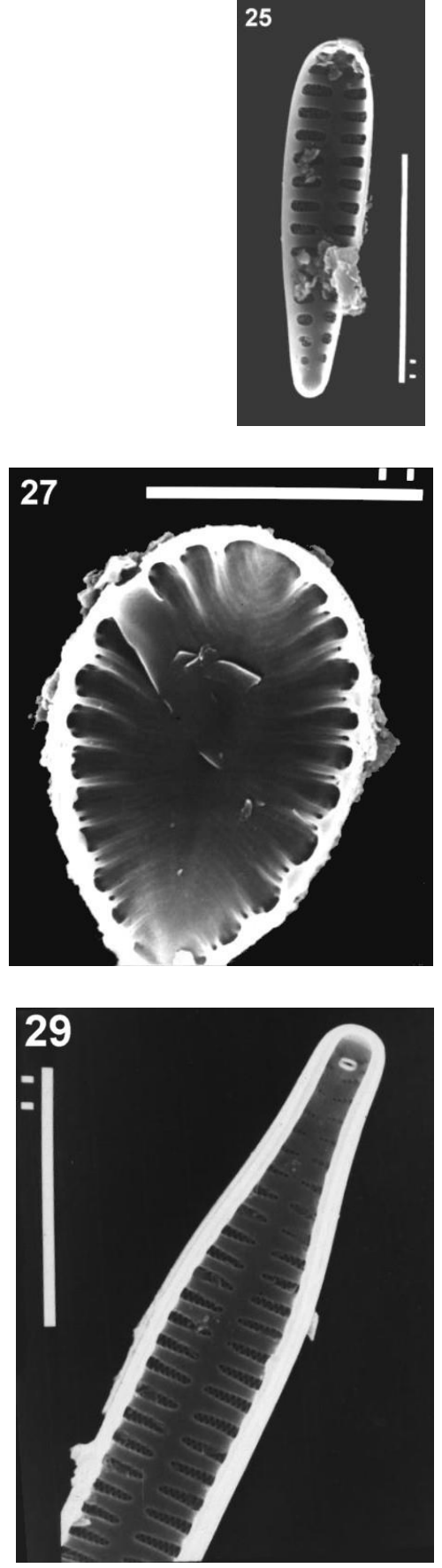

Plate V: Fig. 24: $N$. punctata (W. Sm.) Grun. SEM, bar $=10 \mu \mathrm{m}$, fig. 25: Opephora martyi Herib. SEM, bar $=10 \mu \mathrm{m}$, fig. 26: Surirella ovalis Bréb. SEM, bar $=10 \mu \mathrm{m}$, fig. 27: S. ovata Kütz. SEM, bar $=10 \mu \mathrm{m}$, fig. 28: Synedra S. tabulata (Ag.) Kütz. SEM, bar $=10 \mu \mathrm{m}$, fig. 29: S. ulna var. danica (Kütz.) Grun. SEM, bar $=10 \mu \mathrm{m}$. 